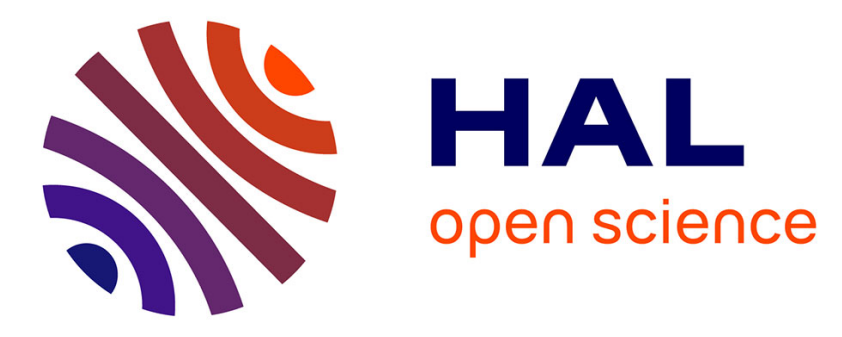

\title{
HIPS recyclé pour la fabrication de composants de télévision
}

\author{
Haritz Eder Ferrando, Laurent Bianchetti, Ferran A Rodríguez, Didac
}

Ferrer-Balas, María Lluïsa Maspoch

\section{- To cite this version:}

Haritz Eder Ferrando, Laurent Bianchetti, Ferran A Rodríguez, Didac Ferrer-Balas, María Lluïsa Maspoch. HIPS recyclé pour la fabrication de composants de télévision. Environnement, Ingénierie \& Développement, 2000, N¹9 - 3ème trimestre 2000, pp.13-16. 10.4267/dechets-sciencestechniques.433 . hal-03181508

\section{HAL Id: hal-03181508 \\ https://hal.science/hal-03181508}

Submitted on 25 Mar 2021

HAL is a multi-disciplinary open access archive for the deposit and dissemination of scientific research documents, whether they are published or not. The documents may come from teaching and research institutions in France or abroad, or from public or private research centers.
L'archive ouverte pluridisciplinaire HAL, est destinée au dépôt et à la diffusion de documents scientifiques de niveau recherche, publiés ou non, émanant des établissements d'enseignement et de recherche français ou étrangers, des laboratoires publics ou privés. 


\section{HIPS RECYCLÉ POUR LA FABRICATION DE COMPOSANTS DE TÉLÉVISION}

Haritz Eder Ferrando, Laurent Bianchetti, Ferran Rodríguez, Didac Ferrer-Balas, Lluïsa Maspoch

* Departamento de Ciencia de los Materiales e Ingeniería Metalúrgica, Centre Català del Plàstic (CCP), Barcelone

Le recyclage des polymères implique souvent des procédés de dégradation thermomécaniques qui ont comme résultat un matériau de qualité souvent médiocre. Dans cet article, une étude des propriétés rhéologiques et mécaniques a été réalisée sur trois échantillons de « Polystyrène choc ", un vierge et deux produits issus du recyclage, en fonction du nombre de cycles successifs de recyclage et de la fraction de matériau recyclé ajouté au matériau vierge.

The recycling of polymers can undergo thermomechanical degradation processes with the results of a poor secondary material, regarding its properties. In this paper an investigation of rheological and mechanical properties of three samples of HIPS (high impact polystyrene, " polystyrene choc ») one virgin and two different recycled, is reported as a function of the number of reprocessing operations and of the fraction of recycled material added to the virgin material.

\section{INTRODUCTION}

Les questions environnementales prennent de plus en plus d'importance dans le monde industriel. Dans le cas des entreprises transformatrices de plastiques, la réduction des résidus générés lors des procédés de transformation est sans aucun doute une préoccupation grandissante. Une fois les technologies de production optimisées, cet objectif est atteint moyennant la récupération des pièces défectueuses et des carottes du cycle d'injection, qui, une fois triturées, peuvent être mélangées avec la matière première alimentant le processus de transformation. Cette technique a pour nom recyclage primaire ou récupération. Après récupération, les plastiques sont soumis à des cycles variés de chauffage et de cisaillement à l'intérieur de la machine de transformation. Ces opérations répétitives conduisent à des dégradations de type thermiques, thermo-oxydatives et mécaniques, avec pour conséquence une diminution des propriétés. Cet effet sera plus ou moins important en fonction de la nature du polystyrène et de sa composition.

Cette étude est centrée sur le HIPS « polystyrène choc ». La consommation de ce matériau pour la fabrication de postes de télévision a atteint, en Espagne, 10700 tonnes en
1997'. L'utilisation des résidus de HIPS provenant de ce secteur a fait l'objet de diverses études antérieures ${ }^{2-7}$. Dans tous les cas étudiés, l'HIPS révèle une grande stabilité (jusqu'à 8 cycles de recyclage à $220^{\circ} \mathrm{C}^{7}$ ) sans variation importante des propriétés, à l'exception de l'élongation à la rupture. Même dans le cas du recyclage de postes de télévision usagés par trituration de l'appareil suivi d'une séparation par flottation, on obtient un HIPS d'une pureté de $96 \%$ avec des propriétés similaires à celles du matériau vierge ${ }^{8}$. Pour notre étude, il s'agit d'effectuer un choix entre deux HIPS recyclés provenant de deux entreprises de recyclage différentes, dans le but de les substituer partiellement ou totalement au matériau vierge. Dans une première partie nous avons choisi le matériau recyclé qui possède les propriétés les plus proches de celles du matériau vierge. Par la suite une étude sur des mélanges de matériau vierge et recyclé en proportion variable a été réalisée. L'influence de plusieurs cycles de transformation sur le matériau recyclé a été étudiée. Un schéma des trois étapes de l'étude est présenté dans la figure I.

\section{MATÉRIELS ET MÉTHODES}

\section{Matériaux}

Le matériau vierge (V) est un HIPS commercial. Les matériaux recyclés (R-A et R-B) sont deux HIPS qui provien-

\section{Tableau I : Explication de la codification utilisée}

\begin{tabular}{ll}
\hline Code & Description du matériau \\
\hline V & HIPS vierge \\
\hline R-A & HIPS recyclé de provenance $A$ \\
\hline$R-B$ & HIPS recyclé de provenance $B$ \\
\hline VE & $V$ extrudé une fois \\
\hline M25E & Mélange de $V$ avec $25 \%$ de $R-A$ extrudé une fois \\
M50E & Mélange de $V$ avec $50 \%$ de R-A extrudé une fois \\
M75E & Mélange de $V$ avec $75 \%$ de R-A extrudé une fois \\
R-AE & R-A extrudé une fois \\
R-AI & R-A récupéré une fois \\
R-A2 & R-A récupéré deux fois \\
E pour matériau extrudé \\
\hline M pour mélange (vierge + recyclé)
\end{tabular}




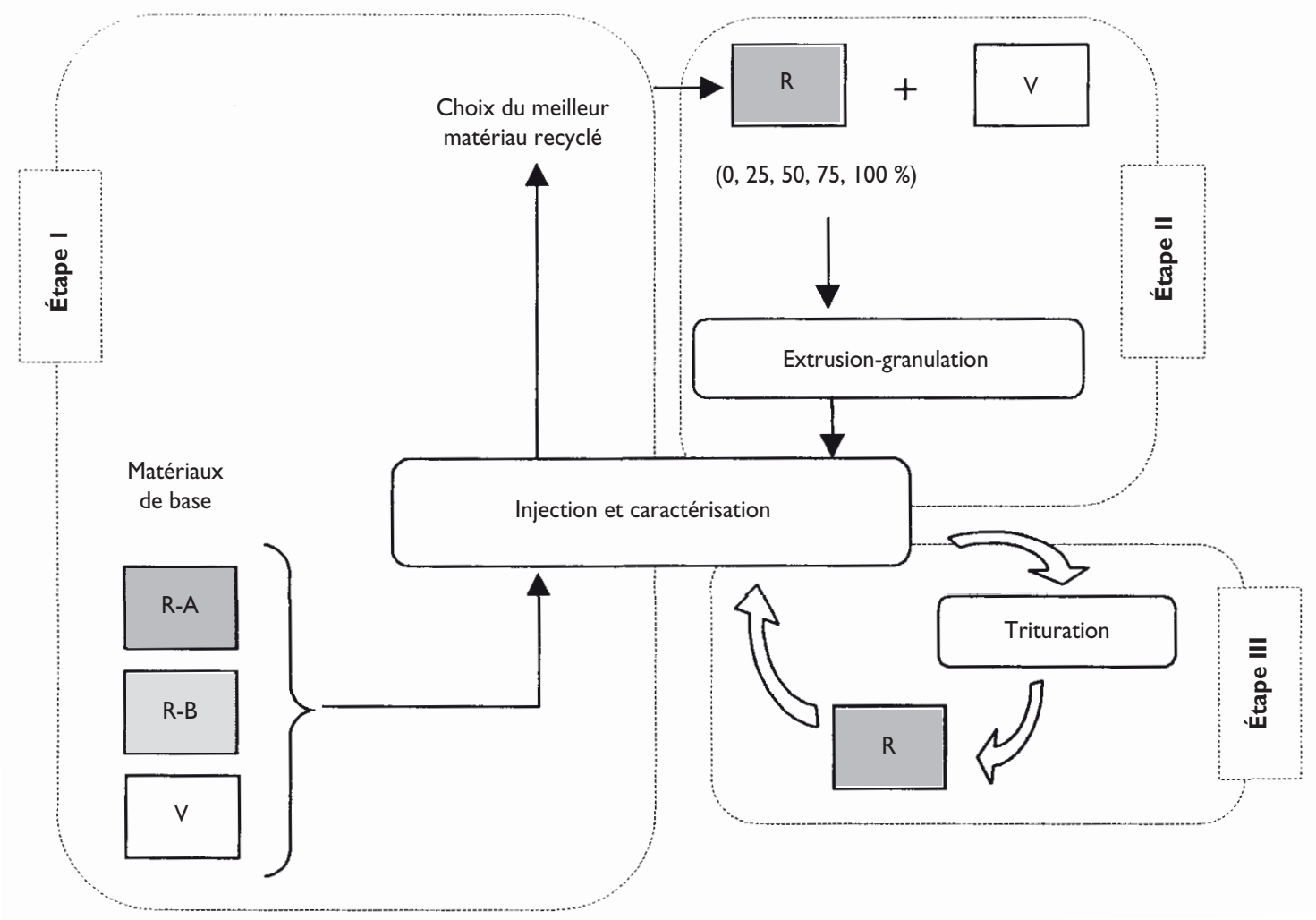

Figure I : Schéma des différentes étapes du projet

nent d'entreprises de recyclage différentes. Ils ont été codifiés comme l'indique le tableau I.

\section{Procédés de transformation}

Les matériaux sont soumis à deux types de procédés de transformation. Pour l'étude des mélanges vierge-recyclé, un premier procédé d'extrusion et de granulation permet l'obtention d'un granulé homogène avant le procédé d'injection. Ceci est effectué à l'aide d'une extrudeuse monovis IQAP LAP E-30/25 D, avec une granulation en fin de ligne. Les éprouvettes sont ensuite obtenues par injection à l'aide d'une machine Mateu \& Solé Meteor 440/90 dans un moule normalisé (figure 4 de la norme ASTM D-647). Pour l'étude de cycles successifs de transformation, les éprouvettes et les carottes d'injection sont triturées dans un moulin Mateu \& Solé I5/2I M 2.6 PM.

\section{Caractérisation}

L'indice de fluidité a été déterminé en utilisant une machine CEAST avec un poids de $5 \mathrm{~kg}$ à une température de $200^{\circ} \mathrm{C}$. Les propriétés mécaniques en traction et en flexion ont été déterminées avec la machine universelle d'essais Adhamel-Logarmy, avec une cellule de charge de $100 \mathrm{kN}$, à température ambiante et avec un conditionnement de $23^{\circ} \mathrm{C}$ et $50 \%$ d'humidité relative pendant 48 heures. Les essais de traction ont été réalisés à une vitesse de $10 \mathrm{~mm} / \mathrm{min}$ et ceux de flexion à une vitesse de $2,8 \mathrm{~mm} / \mathrm{min}$. Les essais d'impact de type Charpy ont été réalisés avec une machine CEAST, un pendule d'une capacité de $2 \mathrm{~J}$ et une vitesse d'impact de $2,9 \mathrm{~m} / \mathrm{s}$ sur des éprouvettes entaillées à une profondeur de 2,5 $\mathrm{mm}$. La détermination de la température de ramollissement Vicat a été réalisée sur une machine Ceast 6505 en appliquant une charge de 49,05 $\mathrm{N}$ et une vitesse de chauffage de $120^{\circ} \mathrm{C} / \mathrm{h}$.

\section{RÉSULTATS ET DISCUSSION}

Pour des raisons de confidencialité, les valeurs numériques portées sur les figures sont arbitraires et ont été normalisées à 100 pour le matériau de référence.

\section{Étude des matériaux de base}

L'examen des résultats obtenus permet de choisir le matériau recyclé le plus adéquat pour réaliser les étapes suivantes de l'étude.

En premier lieu, la microscopie électronique à balayage sur les surfaces de fracture a révélé que les deux matériaux recyclés contiennent des éléments inorganiques (titane et calcium) provenant d'additifs incorporés antérieurement pour leur application spécifique initiale. La présence de ces particules a une influence importante sur les propriétés en traction de R-B, provoquant une forte diminution de l'élongation à la rupture, par rapport au matériau vierge $(\mathrm{V})$. Ceci n'a pas lieu avec le R-A. 


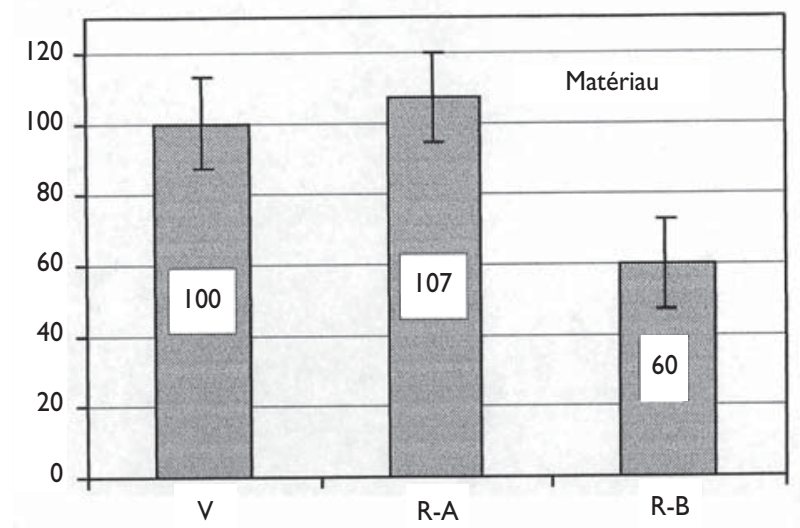

a) Élongation à la rupture (unités arbitraires)

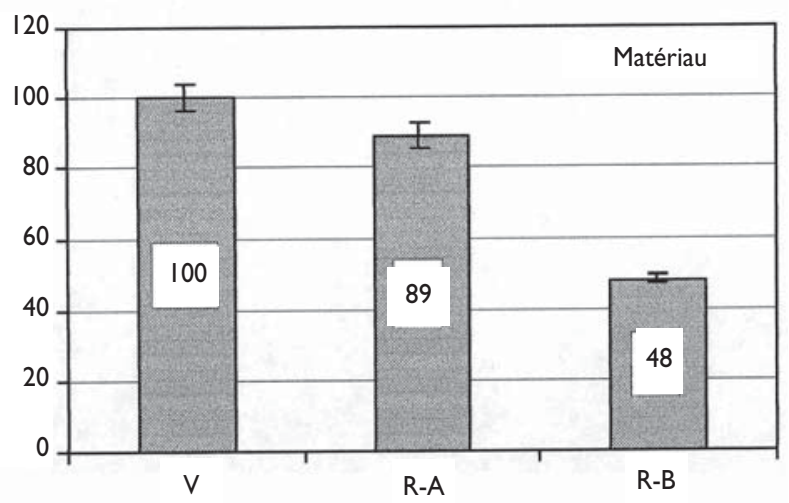

b) Résistance à l'impact Charpy (unités arbitraires)

Figure 2 : Propriétés d'élongation à la rupture (a), et d'impact (b) des matériaux de base

En second lieu, la comparaison des propriétés du $\mathrm{V}$ avec chaque matériau recyclé, montre que :

- $\mathrm{R}$-B se différencie de $\mathrm{V}$ par une fluidité plus grande, ce qui a obligé à injecter les éprouvettes à des conditions différentes de celles de V. II faut donc s'attendre à ce que les deux matériaux aient des difficultés à être mis en œuvre ensemble, dans l'hypothèse d'une utilisation en mélange. On observe également une élongation à la rupture et une résistance à l'impact beaucoup plus basse (figure 2).

- R-A montre un comportement très similaire à celui de $\mathrm{V}$, ceci quelle que soit la propriété étudiée. La résistance à l'impact est la propriété qui varie le plus $\left(-0,9 \mathrm{~kJ} / \mathrm{m}^{2}\right.$, soit II \% inférieure). R-A a été injecté dans les mêmes conditions que $\mathrm{V}$ (contrairement au cas de R-B).

En conclusion de l'étude des matériaux de base, il peut être affirmé que R-A s'impose comme le meilleur matériau recyclé pour substituer $V$ dans cette application et que, par conséquent, l'étude des mélanges vierge-recyclé se fera avec R-A.

\section{Variation des propriétés des mélanges de HIPS vierge et recyclé}

Nous avons préparé trois mélanges avec différentes proportions de matériau recyclé $(25 \%, 50 \%$ et $75 \%$ de R-A ajouté à V). On constate que les trois mélanges possèdent des valeurs semblables pour les propriétés de fluidité, traction, flexion et température de ramollissement. Seule une variation dans la résistance à l'impact a pu être mise en évidence. Pour les trois mélanges les valeurs se trouvent entre celles de $\mathrm{V}$ et celles de R-A, comme on pouvait s'y attendre. Dans la figure 3 nous présentons les variations des valeurs de fluidité et de résistance à l'impact des différents mélanges.

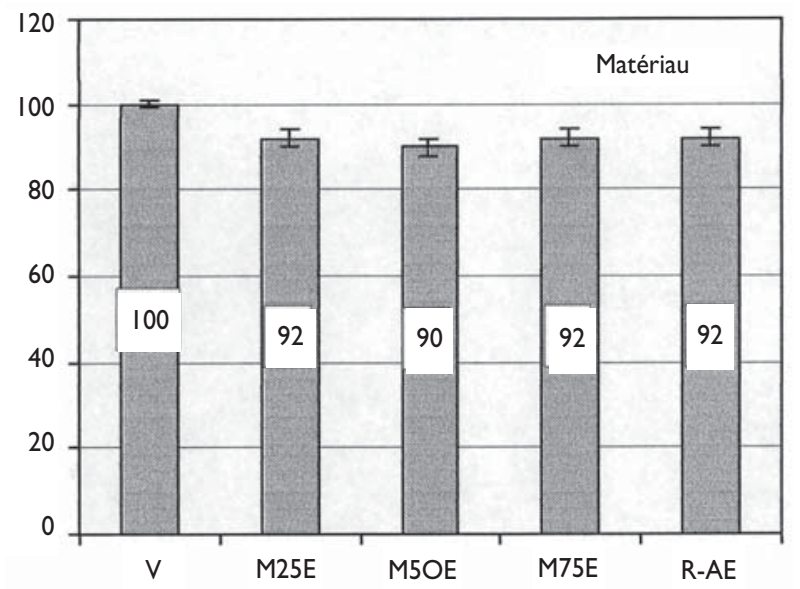

a) MFI (unités arbitraires)

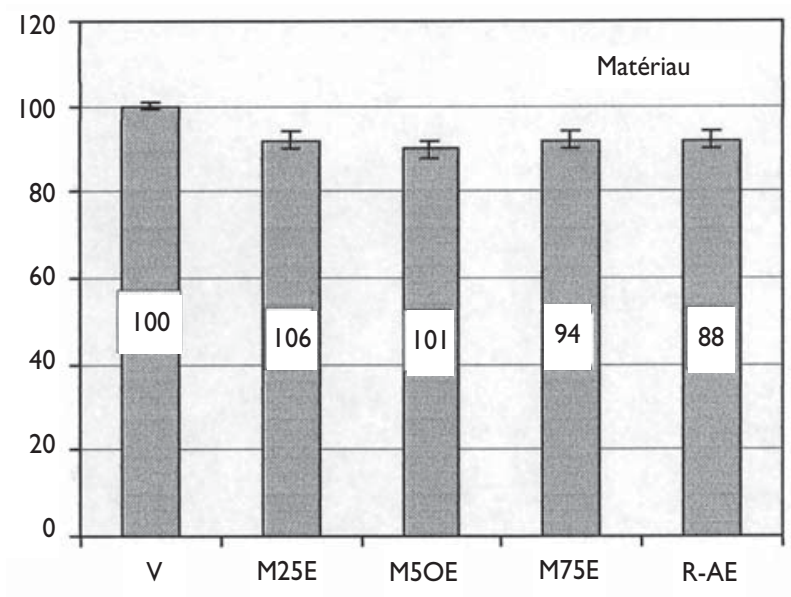

b) Résistance à l'impact C̈harpy (unités arbitraires)

Figure 3 : Propriétés de fluidité (a), et d'impact (b) des mélanges de $\mathbf{V}$ avec $\mathbf{R}-\mathbf{A}$

fluence significative sur les propriétés des mélanges étudiés.

Influence des procédés de transformation successifs sur le matériau recyclé $\mathbf{R}-\mathbf{A}$

En analysant les résultats des essais réalisés sur $R-A$, soumis à plusieurs procédés de transformation successifs, il en ressort les observations suivantes.

Les procédés successifs d'injection (trois fois) et de trituration (deux fois) n'affectent pas la résistance à l'impact, contrairement au cas précédent. En revanche les propriétés de traction et de flexion sont légèrement affectées. Par conséquent, si des composants de télévision devaient être récu- 
pérés à partir de R-A, ces propriétés mécaniques devraient être contrôlées pour assurer la qualité de la pièce finale. L'essai d'indice de fluidité montre que les matériaux R-Al et $\mathrm{R}-\mathrm{A} 2$ ont très peu souffert de la dégradation pendant les procédés d'injection et de trituration. Les résultats des autres essais le confirment, avec peu (ou aucune) variation des propriétés. En augmentant le nombre de cycles de récupération sur le matériau R-A on pourrait le confirmer et déterminer à partir de combien de cycles on observerait une chute importante de ses propriétés mécaniques.

La figure 4 montre, de façon comparative, les variations observées sur les paramètres de flexion, module élastique $\left(E_{f}\right)$ et résistance $\left(\sigma_{f}\right)$.

\section{CONCLUSION}

La qualité d'un matériau recyclé peut varier beaucoup d'un fournisseur à l'autre. Une étude comparative des propriétés mécaniques et rhéologiques permet de choisir le matériau recyclé le plus adapté à chaque application.

La fluidité du HIPS augmente avec la proportion de matériau recyclé, ainsi qu'avec le nombre de procédés de transfor-

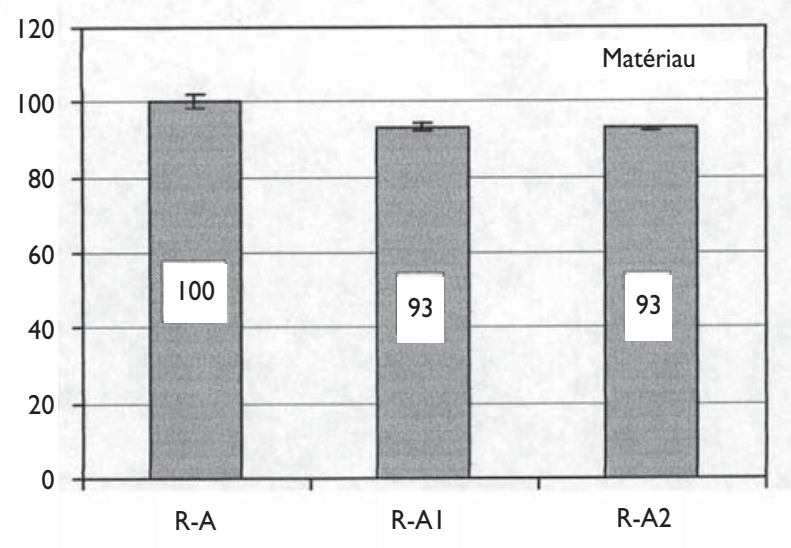

a) Module de flexion (unités arbitraires)

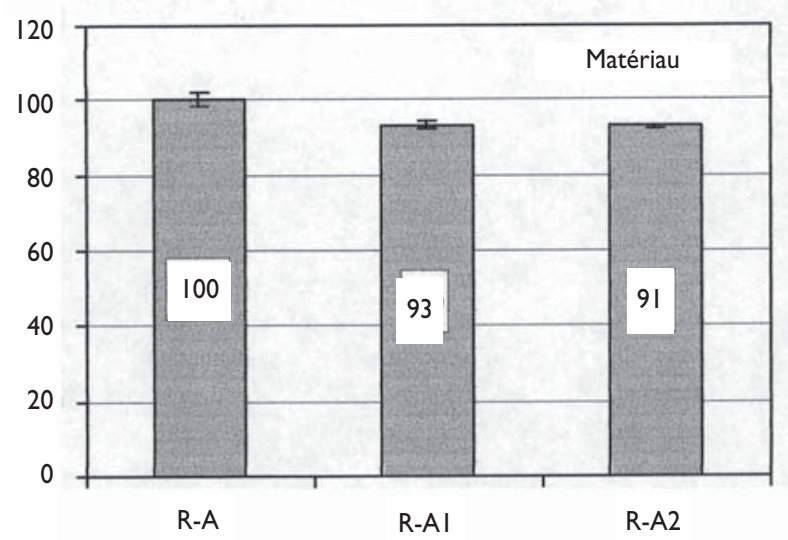

b) Résistance à la flexion (unités arbitraires)

Figure 4 : Variation des propriétés de flexion : (a) module élastique $\mathrm{Ef}$, et $(\mathrm{b})$ résistance of, avec les procédés de transformation successifs sur R-A mation. La propriété mécanique la plus affectée est la résistance à l'impact.

En conclusion on peut affirmer que le matériau recyclé RA peut être substitué totalement au HIPS vierge pour la production de composants de télévision et être récupéré plusieurs fois après le procédé de transformation.

Haritz Eder Ferrando, Laurent Bianchetti, Ferran Rodríguez, Didac Ferrer-Balas, M $^{\mathrm{a}}$ Lluïsa Maspoch* Departamento de Ciencia de los Materiales e Ingeniería Metalúrgica, Centre Català del Plàstic (CCP) - Universitat Politècnica de Catalunya - Avda. Diagonal 647 - 08028 Barcelona (Espagne)

Remerciements

Les auteurs remercient l'entreprise Sony España SA pour le financement et la collaboration dans la réalisation de cette étude, et le Cidem (Centre d'Informació i Desenvolupament Empresarial de la Generalitat de Catalunya) pour son co-financement.

\section{Bibliographie}

I. Centro Español de Plásticos, El sector de los plásticos, 1998.

2. J. Song, L. Suek et M.L. Moran, IEEE, I38-144, 1995

3. Y. Inagaki, M. Kuromiya, T. Noguchi et $H$. Watanabe, IEEE, I2I-124, 1997.

4. F.A. Jamil et S.D.A. Shubber, Plast. Rubb. Proc. Appl., 9, 187, 1988.

5. R.J. Ehrig, Plastics recycling - Products and processes, Hanser, Munich, 1992.

6. J. Brandrup, Recycling and recovery of plastics, Hanser, Munich, 1995.

7. N.K. Kalfoglov et C.E. Chaffey, Polym. Eng. Sci., 19, 552, 1979.

8. E. Langerak, IEEE, 63-68, 1997. 